\title{
Biologie et situation de la Lamproie du Nord, Ichthyomyzon fossor, au Québec
}

\author{
Christian Fortin ${ }^{1}$, Martin Ouellet $^{2}$, Isabelle Cartier ${ }^{1}$, Daniel Banville ${ }^{3}$, et Claude B. Renaud ${ }^{4}$
}

${ }^{1}$ FORAMEC, 70, rue Saint-Paul, Québec, Québec G1K 3V9 Canada; e-mail : christian.fortin@ foramec.qc.ca

${ }^{2}$ Amphibia-Nature, 1902, boulevard de Grande-Grève, Gaspé, Québec G4X 6L6 Canada; e-mail: mouellet@amphibia-nature.org

${ }^{3}$ Ministère des Ressources naturelles et de la Faune, Direction de l'expertise sur la faune et ses habitats, 880, chemin Sainte-Foy, Québec, Québec G1S 4X4 Canada; e-mail: daniel.banville@mrnf.gouv.qc.ca

${ }^{4}$ Musée canadien de la nature, Service de recherche, C.P. 3443, succursale D, Ottawa, Ontario K1P 6P4 Canada; e-mail: crenaud@mus-nature.ca

Fortin, Christian, Martin Ouellet, Isabelle Cartier, Daniel Banville, and Claude B. Renaud. 2007. Biologie et situation de la Lamproie du Nord, Ichthyomyzon fossor, au Québec. Canadian Field-Naturalist 121(4) : 402-411.

La Lamproie du Nord est une espèce propre à l'Amérique du Nord et elle ne se rencontre, au Québec, que dans l'extrême sud de la province. Cette lamproie a été observée dans le fleuve Saint-Laurent ainsi que dans 11 de ses tributaires. Elle aurait disparu de la rivière Yamaska où elle avait été recensée entre 1946 et 1959. Poisson non parasite, sa taille au stade adulte ne dépasse habituellement pas $160 \mathrm{~mm}$. Les larves, appelées ammocètes, et les adultes ne se retrouvent qu'en eau douce. Les cours d'eau utilisés sont généralement des ruisseaux et des rivières à fond graveleux ou sablonneux. La Lamproie du Nord requiert deux habitats distincts, soit un pour les adultes reproducteurs et un autre, le long du même cours d'eau, pour le développement des ammocètes. La détérioration de l'habitat et la pollution des eaux représentent les principales menaces à la survie des lamproies. La faible fécondité et la mobilité réduite de la Lamproie du Nord font en sorte que cette espèce est peu adaptable aux modifications de son environnement.

Mots-clés : Lamproie du Nord, Ichthyomyzon fossor, Petromyzontidae, rapport de situation, Québec.

The Northern Brook Lamprey is endemic to North America. In Quebec, it is restricted to the southernmost part of the province. This lamprey is known from the St. Lawrence River as well as in 11 of its tributaries. It appears to have been extirpated from the Yamaska River where it was collected between 1946 and 1959. It is a nonparasitic fish and its adult size does not usually exceed $160 \mathrm{~mm}$. Larvae, called ammocoetes, and adults are found only in fresh water. Watercourses where it occurs are generally streams and rivers with gravel or sandy bottoms. The Northern Brook Lamprey requires two types of habitats during its life cycle, one for spawning adults and one, along the same watercourse, for the developing ammocoete. Habitat degradation and water pollution are the major threats to the survival of lampreys. The low fecundity and low dispersal abilities of the Northern Brook Lamprey make this species poorly adapted to withstand changes in its environment.

Key Words: Northern Brook Lamprey, Ichthyomyzon fossor, Petromyzontidae, status report, Québec.

Le genre Ichthyomyzon comprend six espèces qui se regroupent en trois paires, chacune étant composée d'une espèce parasite (espèce souche) et d'une espèce non parasite (espèce satellite; Vladykov et Kott 1979a). La Lamproie du Nord (Ichthyomyzon fossor), une espèce non parasite, serait issue de la Lamproie argentée (Ichthyomyzon unicuspis), une espèce parasite (Vladykov et Kott 1979a). Toutefois, certaines analyses génétiques récentes ont remis en question le fait que la Lamproie du Nord soit vraiment distincte de la Lamproie argentée (COSEPAC 2007*). La Lamproie du Nord est un petit poisson d'eau douce. Au stade larvaire, les larves, que l'on désigne sous le terme d'ammocètes, vivent enfouies dans les sédiments et se nourrissent par filtration. Au stade adulte, une fois la métamorphose terminée, l'espèce ne conserve pas de tractus intestinal fonctionnel et ne peut ainsi se nourrir. $\mathrm{Au}$ cours de sa vie, elle se reproduit seulement une fois et meurt peu de temps après la fraie.

L'aire de répartition de la Lamproie du Nord est limitée à l'Amérique du Nord. Au Canada, on la retrouve au Manitoba, en Ontario et au Québec. Elle a été identifiée au Québec comme espèce susceptible d'être désignée «menacée ou vulnérable ». Au Canada, la Lamproie du Nord n'est pas encore officiellement désignée, mais elle possède le statut d'espèce «préoccupante » attribué par le Comité sur la situation des espèces en péril au Canada (COSEPAC). Un rapport de situation, commandé par cet organisme, a été réalisé en 1991 (Lanteigne 1991*) et une mise à jour a été effectuée en 2007 (COSEPAC 2007*). Le présent article constitue une mise à jour du rapport de situation officiel soumis au ministère des Ressources naturelles et de la Faune du Québec (Fortin et al. $\left.2005^{*}\right)$.

\section{Description}

La taxinomie des lamproies holarctiques au stade adulte se fonde principalement sur quatre caractères, soit la disposition des dents, le nombre de myomères du tronc, les proportions du corps et la répartition géographique de l'espèce (Vladykov et Kott 1979b*). La longueur totale de la Lamproie du Nord adulte ne dépasse habituellement pas $160 \mathrm{~mm}$ (94 à $146 \mathrm{~mm}$ : 


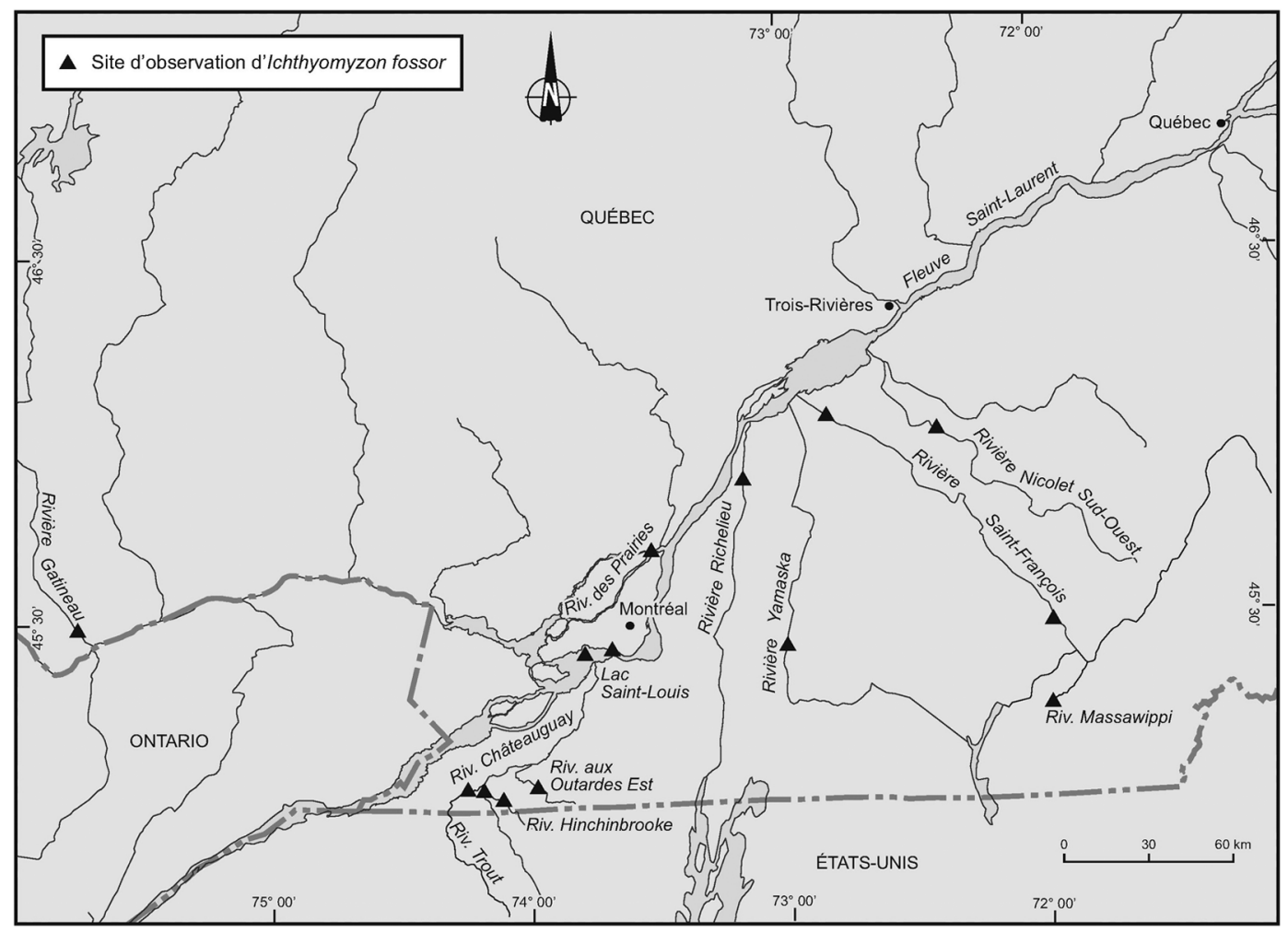

FIGURE 1. Répartition québécoise de la Lamproie du Nord, Ichthyomyzon fossor.

Hubbs et Trautman 1937; 128 à $150 \mathrm{~mm}$ : Vladykov 1952; 86 à 166 mm: Morman 1979*; 98 à 158 mm: Lanteigne 1981; 130 à 173 mm: Trautman 1981). Son corps est de forme cylindrique et sa tête est courte et dépourvue de mâchoire. Elle possède une bouche ronde en forme de ventouse dont l'ouverture est toujours plus courte que la longueur de la région branchiale. Elle est pourvue de sept paires de branchies. Les yeux sont petits et situés près du haut de la tête. Le nombre de myomères du tronc varie généralement entre 50 et 53 (Lanteigne 1988).

Les dents de l'entonnoir buccal sont peu développées. La lamina supraorale consiste en une dent pourvue de deux pointes alors que la lamina linguale transversale possède entre six et onze petites pointes sur l'arête denticulée (Scott et Crossman 1974). Les dents latérales internes consistent quant à elles en quatre dents unicuspides situées sur chaque côté du disque. Des dents latérales externes et de petites pointes situées dans les champs antérieur et postérieur complètent la dentition. La peau est lisse et ne possède pas d'écailles. L'adulte est bicolore : coloration gris foncé à brune sur le dos et les flancs et grise à blanc argenté sur les parties inférieures (Vladykov 1949*). La surface ventrale a une teinte orangée, particulièrement chez les femelles sexuellement matures (Leach 1940). Le repli anal est plus visible chez la femelle sexuellement mature que chez le mâle et est fusionné à la nageoire caudale (Scott et Crossman 1974). La nageoire dorsale est continue, ce qui la différencie de la Lamproie de l'Est (Lampetra appendix).

\section{Répartition}

La Lamproie du Nord est une espèce propre à l'Amérique du Nord. Au Canada, elle se retrouve dans le bassin hydrographique de la baie d'Hudson, au Manitoba, et dans le bassin hydrographique des Grands Lacs et du fleuve Saint-Laurent, en Ontario et au Québec (Lanteigne 1992). Aux États-Unis, elle se rencontre dans le bassin du Mississippi depuis le Wisconsin jusqu'à l'Ohio et vers le sud jusqu' au Kentucky (Lanteigne 1992). Il existe aussi une population disjointe au Missouri.

La connaissance de la répartition de la Lamproie du Nord au Québec est très restreinte. Elle se limite à des mentions rapportées dans le cadre de travaux de recherches et d'inventaires multispécifiques. Cette espèce n'a jamais fait l'objet d'un programme d'inventaire systématique et ciblé au Québec, à l'exception des travaux de Vladykov (1952) dans les années 1940 et 1950 . Le manque de données sur sa répartition réside, entre autres, dans la nature discrète de ses mœurs tant au stade larvaire qu'adulte, à une faible longévité des individus une fois métamorphosés, à 
leur petite taille, à la difficulté d'identifier les lamproies à l'espèce et dans le manque d'inventaires spécifiques. Les populations actuellement connues sont localisées dans le Québec méridional (Figure 1). Elles se retrouvent uniquement en eau douce dans le fleuve SaintLaurent et dans le cours de certaines rivières tributaires du Saint-Laurent (Tableau 1). Des inventaires ciblés pourraient permettre de confirmer la présence de l'espèce ailleurs dans le fleuve Saint-Laurent et dans certains autres de ses tributaires.

La Lamproie du Nord a déjà été observée en grand nombre dans la rivière Yamaska (près de Saint-Césaire) entre 1946 et 1950 (Vladykov 1952). Malgré un effort intensif au moyen de la pêche électrique, aucun spécimen d'ammocète n'a été observé lors de travaux réalisés en 1990 (Renaud et al. 1995a) et 1992 (C. B. Renaud, non publié) à cette localité. Des quatre stations où Vladykov (1952) a trouvé des spécimens, le site de Saint-Césaire était qualifié d'endroit par excellence pour la Lamproie du Nord. L'espèce pourrait s'être maintenue dans la rivière Saint-François, près de Pierreville, où elle fut observée en 1947, 1951 et 1990. Vladykov (1952) qualifie ce site d'habitat idéal pour les ammocètes, en raison de l'abondance des îles et du substrat de fond favorable. La Lamproie du Nord a de nouveau été observée dans cette rivière en 2003, mais cette fois dans la localité de Bromptonville, à environ $130 \mathrm{~km}$ en amont de Pierreville (Desroches et al. 2008).

\section{Biologie générale \\ Cycle vital}

Le cycle vital des lamproies comprend deux stades : le stade larvaire (ammocète) et le stade adulte. Tôt après l'éclosion, la jeune ammocète émerge du nid et migre passivement vers l'aval. Après avoir trouvé un habitat favorable, elle creuse un trou en forme de U dans un substrat plutôt mou où elle demeure généralement cachée. Les ammocètes sont en effet des animaux fouisseurs relativement sédentaires (Potter 1980). Ce stade larvaire persiste de trois à six ans chez la Lamproie du Nord (Hardisty et Potter 1971a). Une fois qu'elle a atteint une certaine taille (moyenne de $131 \mathrm{~mm}$ : Leach 1940; moyenne de $114 \mathrm{~mm}$ : Purvis 1970), la larve se métamorphose entre les mois d'août et novembre. Le début de la métamorphose est synchrone entre les individus d'une même population; un accroissement rapide et substantiel de la température serait un des facteurs impliqués dans l'initiation de ce phénomène (Potter 1980). D’une durée de 2 à 3 mois, la métamorphose est un lent processus au cours duquel l'individu cesse de se nourrir (Leach 1940). On observe même une réduction de la longueur totale des individus au cours de cette phase. La bouche se transforme en un entonnoir buccal pourvu de dents. Cette période est suivie d'un stade adulte immature qui se déroule généralement entre novembre et février et au cours duquel l'individu est semi-sédentaire (Leach 1940). Une période plus active menant à la maturité sexuelle a lieu de février à mai. L'adulte ne se nourrit jamais. La fraie survient généralement en mai et est suivie quelques jours plus tard par la mort des individus reproducteurs.

\section{Alimentation}

Le régime alimentaire des ammocètes se compose principalement de détritus organiques, auxquelles s'ajoutent des algues unicellulaires (principalement des diatomées) et des bactéries (Sutton et Bowen 1994). Les ammocètes utilisent efficacement les détritus organiques pendant les mois les plus chauds lorsque la température des cours d'eau et la qualité des aliments sont plus favorables à l'alimentation, la digestion et la croissance (Sutton et Bowen 1994). Elles se nourrissent très lentement, ce qui leur permettrait d'utiliser au maximum une ressource de faible qualité. Leur taux d'alimentation est d'ailleurs consistant avec le faible métabolisme observé chez ce groupe de poissons (Hill et Potter 1970). Le taux d'alimentation dépend de la température et, à un degré moindre, de la densité des aliments (Moore et Mallatt 1980). La variation dans l'abondance des algues et des bactéries dans l'alimentation des ammocètes correspond à leur disponibilité dans les cours d'eau durant le cycle annuel (Sutton et Bowen 1994). Les bactéries et les algues ne peuvent cependant supporter à elles seules la croissance et le métabolisme des larves; les détritus organiques sont nécessaires à ces fonctions (Moore et Mallatt 1980; Sutton et Bowen 1994). La Lamproie du Nord adulte, tout comme les autres espèces de lamproies non parasites, ne conserve pas de tractus intestinal fonctionnel une fois la métamorphose complétée. Par conséquent, elle ne peut pas manger et n'atteint jamais la taille de l'espèce parasite à laquelle elle est apparentée, soit la Lamproie argentée (Vladykov et Kott 1979b*).

\section{Reproduction}

La Lamproie du Nord fraie uniquement en eau douce et atteint généralement l'âge de reproduction à six ans. Les lamproies ne pondent qu'une seule fois dans leur vie et tous les adultes meurent quelques jours après la fraie (Leach 1940). La température de l'eau est le facteur critique qui détermine le début de la période de reproduction chez les lamproies (Hardisty et Potter 1971b; Manion et Hanson 1980). Au Québec, la reproduction a lieu en mai, avec un pic de fraie à des températures de l'eau entre 13 et $16^{\circ} \mathrm{C}$ (Vladykov 1949*). Comtois et al. (2004) ont capturé une femelle en fraie un 23 juin dans la rivière Gatineau à une température de $19^{\circ} \mathrm{C}$. Au Michigan, le pic de reproduction s'étend de la fin mai à la mi-juin, à une température de l'eau moyenne de $18^{\circ} \mathrm{C}$ (Morman 1979*). Toujours au Michigan, Reighard et Cummins (1916) rapportent une température optimale de l'eau entre 20 et $22^{\circ} \mathrm{C}$; la fraie était rarement observée à des températures inférieures à $18^{\circ} \mathrm{C}$. Elle aurait lieu vers la fin mai dans la rivière Tippecanoe, en Indiana (Leach 1940). 
TABLEAU 1. Liste des mentions d'observations de la Lamproie du Nord, Ichthyomyzon fossor, au Québec.

\begin{tabular}{llcl}
\hline \hline Cours d'eau & Localité & Année d'observation & Source \\
\hline Fleuve Saint-Laurent & Rapides de Lachine & 1950 & Vladykov 1952 \\
Lac Saint-Louis & Pointe-Claire & 1941 & CDPNQ \\
Rivière Châteauguay & Athelstan & 1990,1992 & Renaud et al. 1995b, 1998 \\
Rivière des Prairies & Lachenaie & 1998 & CDPNQ \\
Rivière Gatineau & Gatineau & 1999 & Comtois et al. 2004 \\
Rivière Hinchinbrooke & Hinchinbrooke & 1976 & MRNF $^{\text {b }}$ \\
Rivière Massawippi & North Hatley & 1995 & Desroches et al. 2008 \\
Rivière Nicolet Sud-Ouest & Mitchell Station & 1951 & Vladykov 1952 \\
Rivière aux Outardes Est & Franklin & 2002 & J. Dubé, communication personnelle \\
Rivière Richelieu & Saint-Roch-de-Richelieu & 1990 & Renaud et al. 1995b \\
Rivière Saint-François & Pierreville & 1947,1951 & Vladykov 1952 \\
& Pierreville & 1990 & Renaud et al. 1995a \\
Rivière Trout & Bromptonville & 2003 & Desroches et al. 2008 \\
Rivière Yamaska & Huntingdon & 1976 & CDPNQ \\
& Saint-Césaire & $1946-1950$ & Vladykov 1952 \\
\hline \hline
\end{tabular}

a Centre de données sur le patrimoine naturel du Québec.

b Ministère des Ressources naturelles et de la Faune du Québec. Direction régionale de la Montérégie. Fichier informatisé des relevés fauniques en milieu aquatique et riparien.

En plus de la température de l'eau, deux autres facteurs sont essentiels au succès de ponte, soit un substrat adéquat et un courant uniforme et unidirectionnel s'écoulant au-dessus du nid (Manion et Hanson 1980; Lanteigne 1992). La Lamproie du Nord fraie dans des ruisseaux et des petites rivières sur fond de gros gravier, de galets ( 25 à $152 \mathrm{~mm}$ de diamètre) et de sable (Reighard et Cummins 1916; Scott et Crossman 1974). Bien que la fraie des lamproies soit généralement associée à des habitats peu profonds sans couvert de protection, il est maintenant admis que les espèces du genre Ichthyomyzon se reproduisent aussi sous un couvert de grosses roches ou de débris ligneux (Cochran et Gripentrog 1992). Ce comportement permettrait aux lamproies de se reproduire dans des milieux où l'eau est plus profonde et où le courant serait autrement trop fort pour frayer dans des milieux ouverts. Il réduirait aussi la vulnérabilité des reproducteurs à certains types de prédateurs (Cochran et Gripentrog 1992). Hardisty et Potter (1971b) rapportent que les cours d'eau typiques des lamproies de ruisseaux ont une vitesse du courant située entre 0,3 et $0,5 \mathrm{~m} / \mathrm{s}$ dans les aires de fraie.

Les adultes ne semblent pas migrer en masse vers les aires de fraie (Leach 1940). Une fois arrivés sur les lieux, les reproducteurs se regroupent habituellement dans une aire restreinte (Scott et Crossman 1974). Les mâles amorcent alors la nidification (Manion et Hanson 1980; Moyle et Cech 2004). Ils établissent leurs nids en déplaçant de petits cailloux à l'aide de leur bouche afin de créer une légère dépression. Ils déplacent ensuite le sable à l'aide de mouvements vigoureux du corps (Hardisty et Potter 1971b). Le nid a un diamètre d'environ 7 à $10 \mathrm{~cm}$ (Scott et Crossman 1974). La profondeur se situe généralement entre 20 et $46 \mathrm{~cm}$, de la surface de l'eau jusqu'au fond du nid
(Hardisty et Potter 1971b; Scott et Crossman 1974). Leach (1940) rapporte qu'une femelle pond environ 1195 œufs, mais cette donnée n'est basée que sur un échantillon composé de deux femelles. Pour sa part, Vladykov (1951) mentionne une moyenne de 1524 œufs (1115 à 1979), à partir de neuf spécimens récoltés au Québec. Le diamètre moyen d'un œuf se situe entre 1,0 et 1,2 mm (Vladykov 1951; Scott et Crossman 1974). Après la fertilisation, les œufs sont recouverts du substrat environnant le nid (Hardisty et Potter 1971b). Les oeufs éclosent 2 à 4 semaines plus tard (Leach 1940).

\section{Déplacements}

La répartition des lamproies le long d'un cours d'eau est le résultat de deux types de comportements liés aux déplacements des adultes et des larves. Le premier correspond à la migration vers l'amont des adultes reproducteurs aux aires de fraie. La migration des lamproies non parasites se ferait sur des distances beaucoup plus courtes que chez les espèces parasites (Hardisty et Potter 1971b). Le deuxième type de déplacements concerne la migration des ammocètes vers l'aval, là où les conditions d'envasement et de courant sont plus favorables à l'alimentation et à l'enfouissement dans le substrat. Cette migration est en partie passive (Potter 1980). Le déplacement des ammocètes est fonction de leurs mouvements, des conditions hydrographiques et hydrologiques locales, de la température de l'eau, de la saison, de la stabilité et de la nature du fond du cours d'eau, de la qualité de l'eau et de leur densité en relation avec la qualité de l'habitat (Hardisty et Potter 1971a; Morman et al. 1980; Potter 1980). Certains auteurs suggèrent que cette migration est minimale, tout particulièrement dans les cours d'eau où les gradients sont faibles, où l'écoulement est stable et où les habitats sont propices 
(Morman et al. 1980). Elle pourrait cependant être plus importante dans les cours d'eau où les gradients sont plus importants, les ammocètes plus âgées étant de plus en plus prédominantes vers l'aval (Hardisty et Potter 1971a; Potter 1980). Bien que les ammocètes nagent relativement bien, il est improbable qu'elles puissent soutenir cette grande vitesse au cours d'une longue période (Potter 1980). Elles peuvent cependant nager à contre-courant, si ce dernier est faible (Hardisty et Potter 1971a). Les déplacements volontaires des ammocètes s'effectueraient principalement la nuit (Potter 1980), ce qui réduirait l'impact des prédateurs diurnes. La migration vers l'aval des individus métamorphosés se réalise aussi généralement la nuit et est influencée par le débit et la température du cours d'eau (Potter 1980).

\section{Parasites et maladies}

À notre connaissance, aucune étude n'a décrit la nature et la fréquence des parasites et des maladies spécifiques à la Lamproie du Nord. L'espèce est probablement susceptible à un bon nombre de maladies non spécifiques que l'on retrouve chez les autres poissons d'eau douce du Québec (Uhland et al. 2000). Chez la Lamproie marine (Petromyzon marinus), Wilson et Ronald (1967) ont observé que les adultes capturés en lac avaient un taux d'infection parasitaire (19\%) beaucoup plus faible que les spécimens qui retournaient en rivière pour frayer $(53 \%)$.

\section{Longévité}

Chez les lamproies, la durée du stade adulte est beaucoup plus courte que celle du stade larvaire. Dans le cas de la Lamproie du Nord, les adultes ne vivent qu'environ 6 mois, tandis que les ammocètes ont besoin de 3 à 6 ans pour arriver à leur métamorphose, selon le lieu et la nourriture (Okkelberg 1922; Leach 1940; Purvis 1970). L'âge maximum d'un adulte serait donc d'au plus 7 à 8 ans. La durée précise du stade larvaire est difficile à déterminer avec assurance car l'estimation est basée sur des classes de fréquence de longueur totale des individus (Leach 1940; Scott et Crossman 1974; Potter 1980).

\section{Habitats}

La Lamproie du Nord habite généralement les ruisseaux et les rivières à fond graveleux ou sablonneux (Jyrkkanen et Wright 1979; Morman 1979*). Au Québec, on la retrouve aussi dans le fleuve SaintLaurent. Elle semble éviter les eaux stagnantes et les lacs (Hubbs et Trautman 1937). Dans le bassin hydrographique du lac Supérieur, Schuldt et Goold (1980) ont observé la Lamproie du Nord dans des cours d'eau de taille intermédiaire où le débit moyen estival variait de 0,3 à $28,3 \mathrm{~m}^{3} / \mathrm{s}$; l'espèce était commune dans plusieurs cours d'eau aux eaux turbides et habitait les régions de la tête des eaux. On ne retrouve la Lamproie du Nord qu'en eau douce; elle ne tolère aucunement la salinité (Vladykov 1949*). La rivière Yamaska, près de Saint-Césaire, représentait un habitat typique de la Lamproie du Nord selon Vladykov (1952).

En fonction de l'âge des individus, la Lamproie du Nord requiert deux habitats distincts le long d'un cours d'eau donné, soit un pour les adultes reproducteurs et un autre pour le développement des ammocètes. Les adultes reproducteurs ont besoin de petits cours d'eau aux eaux claires et à gradient topographique élevé, avec présence de rapides et un fond de gravier et de sable (Trautman 1981). Quant aux ammocètes, elles préfèrent la portion des cours d'eau à courant plus lent, là où le substrat est propice à l'enfouissement (substrat mou; Potter et al. 1986). Ce substrat renferme souvent un contenu élevé en matériel organique (Hardisty et Potter 1971a; Potter et al. 1986; Beamish et Lowartz 1996). La composition des sédiments, dont la taille des particules ainsi que l'hétérogénéité, constitue un facteur important dans la sélection de l'habitat (Hardisty et Potter 1971a; Anderson et White 1988; Beamish et Lowartz 1996). Les ammocètes éviteraient les fonds composés principalement de très petites ou de grosses particules, le fond étant trop compact dans le premier cas et les particules trop lourdes pour être délogées par les larves dans le second (Beamish et Lowartz 1996). La taille idéale correspondrait à du sable mi-fin.

Au Michigan, Morman (1979*) a observé que la Lamproie du Nord était plus tolérante à l'eau chaude que les autres espèces étudiées. Les plus grandes densités d'ammocètes se retrouvaient habituellement dans les sections les plus chaudes des cours d'eau. Ce type de milieux serait généralement non propice à la présence de l'Omble de fontaine (Salvelinus fontinalis; Vladykov 1949*). Les ammocètes sont photophobiques, ce qui expliquerait pourquoi l'ombre est un facteur qui influence significativement leur répartition (Potter et al. 1986). La quantité de chlorophylle $a$ à la surface du substrat serait aussi une variable environnementale importante expliquant la densité des larves de lamproie (Potter et al. 1986). Cette observation reflète bien l'importance des diatomées et autres microalgues dans le régime alimentaire des ammocètes qui évitent généralement les eaux stagnantes ou trop riches en matière organique, là où la teneur en oxygène est faible (Potter 1980).

\section{Dynamique des populations}

Les connaissances sur la dynamique des populations de la Lamproie du Nord sont limitées. La fécondité de cette espèce fut caractérisée au Québec par Vladykov (1951) et est considérée faible par rapport aux autres espèces de lamproies étudiées. La survie des œufs dans les nids n'a pas été déterminée chez la Lamproie du Nord, mais chez la Lamproie marine, celle-ci est élevée, pouvant atteindre $90 \%$ (Manion et Hanson 1980). À partir d'un échantillon de 627 larves de Lamproie du Nord, Purvis (1970) a observé un rapport des sexes de 1:1 dans un tributaire du lac Supérieur, au Michigan. Chez les adultes reproduc- 
teurs, il semble que le rapport des sexes favorise les mâles (75 \%, Purvis 1970; $71 \%$, Jyrkkanen et Wright 1979). Ce phénomène est observé chez plusieurs autres espèces de lamproies non parasites (Potter 1980).

Chez la Lamproie du Nord, comme chez d'autres espèces de lamproies, une classe d'âge peut contribuer à plusieurs cohortes de reproducteurs. En effet, seule une fraction d'une classe d'âge se métamorphose une année donnée, les autres individus de la même classe se métamorphosant au cours des années suivantes (Purvis 1970). Ce recrutement de différentes classes d'âge à la population reproductrice pourrait expliquer les fluctuations de populations observées chez la Lamproie de Planer (Lampetra planeri), une espèce européenne (Hardisty 1961). La température de l'eau et la disponibilité de nourriture seraient des facteurs déterminants pour la croissance des ammocètes (Hardisty et Potter 1971a). La croissance variait beaucoup entre les classes d'âge étudiées par Purvis (1970). L'auteur suggère que cette différence est principalement causée par une forte diminution de la densité de la population consécutive à des traitements chimiques avec des lampricides. Il en résulterait une diminution de la compétition pour la nourriture et l'espace et une augmentation de la croissance dans la population résiduelle.

\section{Pressions sur l'espèce, facteurs limitants et adaptabilité}

La détérioration de l'habitat constitue probablement la principale pression sur l'espèce (Vladykov 1973; Renaud 1997). Différents types de pollution, seule ou en combinaison avec d'autres facteurs, restreignent la répartition des lamproies (Morman et al. 1980; Renaud 1997). La pollution de l'eau liée au développement agricole semble être la principale cause de la disparition apparente de la Lamproie du Nord de la rivière Yamaska, au niveau de Saint-Césaire (Renaud et al. 1995a). Les programmes de contrôle chimique de la Lamproie marine introduite dans les bassins hydrographiques des lacs Huron, Michigan et Supérieur (Smith et Tibbles 1980) ont affecté les populations locales d'autres espèces de lamproies, dont la Lamproie du Nord (Schuldt et Goold 1980). Les larves de cette espèce ainsi que de la Lamproie argentée ont disparu de 41 des 81 cours d'eau traités du bassin hydrographique du lac Supérieur qu'elles fréquentaient jadis, tant au Canada qu'aux États-Unis (Schuldt et Goold 1980). Le lampricide utilisé, le 3-trifluorométhyl-4nitrophénol (TFM), est destiné à tuer les larves avant leur métamorphose. Le genre Ichthyomyzon serait de susceptibilité intermédiaire au TFM (Davis 1970).

La Lamproie du Nord était utilisée autrefois comme appât pour la pêche sportive (Vladykov 1973). Vladykov (1952) mentionne des récoltes totales annuelles d'environ 300000 ammocètes pour l'ensemble du Québec dans les années 1940 et 1950, toutes espèces confondues. L'impact qu'a eu cette pratique sur les populations québécoises est cependant inconnu. Cette utilisation pour la pêche sportive est maintenant interdite au Québec. L'accumulation de sédiments transportés par l'eau jusqu'aux aires de fraie, les barrages et autres obstacles bloquant les remontées d'adultes reproducteurs et l'abaissement du niveau de l'eau, lesquels constituent une menace pour la survie des larves dans leurs abris, représentent d'autres pressions sur l'espèce (Scott et Crossman 1974; Morman et al. 1980; Lanteigne 1992; Renaud 1997).

La mortalité peut parfois être élevée au tout début du cycle vital (Potter 1980). Diverses espèces de poissons ont en effet été observées en train de se nourrir d'œufs de lamproies ou de très jeunes larves (Hardisty et Potter 1971a; Vladykov 1973; Potter 1980; Lanteigne 1992). Les jeunes larves sont aussi susceptibles à la prédation lorsqu'elles quittent le nid et migrent vers l'aval (Hardisty et Potter 1971a; Potter 1980). La prédation sur les ammocètes plus âgées ne semble cependant pas être un facteur de mortalité important car elles demeurent enfouies (Potter 1980). Trautman (1981) considère les ammocètes et les adultes comme très vulnérables à la prédation par d'autres poissons. Par ailleurs, des cas de prédation sur les adultes reproducteurs ont été rapportés pour une panoplie de prédateurs tels que belette, chien domestique, couleuvre d'eau, goéland, héron, rat musqué, raton laveur et renard (Morman et al. 1980). Les lamproies au nid sont davantage susceptibles à la prédation, car elles sont plus exposées dans des eaux relativement peu profondes.

La compétition interspécifique, les parasites et les maladies ne semblent pas influencer de façon importante la répartition et l'abondance de la Lamproie marine (Morman et al. 1980). L'importance de ces derniers facteurs pour la Lamproie du Nord reste inconnue. La faible fécondité de la Lamproie du Nord suggère qu'il pourrait être plus difficile pour elle de se rétablir à la suite d'un déclin des populations (Schuldt et Goold 1980; Lanteigne 1992). Aussi, la mobilité restreinte de l'espèce limite ses chances de pouvoir recoloniser les cours d'eau où elle aurait disparu. Ces éléments font de la Lamproie du Nord une espèce peu adaptable aux modifications de son environnement.

\section{Importance particulière de l'espèce}

Même si les lamproies constituent un mets de choix dans certaines régions hors Québec (Moyle et Cech 2004), ce type de poissons est actuellement sans grande valeur économique. N'étant pas un poisson parasite, la Lamproie du Nord ne présente pas le danger de blesser ou de tuer d'autres espèces de poissons, au contraire de la Lamproie marine par exemple. La Lamproie du Nord présente cependant un intérêt certain d'ordre scientifique et écologique. Cette espèce fait en effet partie d'un groupe de poissons parmi les plus primitifs (Forey et Janvier 1993) et offre ainsi l'occasion d'étudier l'évolution des poissons et les raisons pour lesquelles les lamproies ont continué à se maintenir dans un environnement en perpétuel change- 
ment (Lanteigne 1992; Moyle et Cech 2004). Les lamproies sont également utiles comme animaux de laboratoire, particulièrement en neurobiologie (Moyle et Cech 2004). Les ammocètes filtrent différents types de micro-organismes et constituent une source de nourriture pour plusieurs espèces de poissons, d'où leur importance dans la chaîne alimentaire (Vladykov 1973). La présence de ce poisson dans le Québec méridional contribue aussi à la biodiversité des ruisseaux et des rivières. Comme les ammocètes sont des poissons relativement sédentaires passant une bonne partie de leur vie enfouis dans les sédiments, elles représentent ainsi des bio-indicateurs potentiels des niveaux de contaminants persistants dans les milieux d'eau douce. Une étude réalisée au Québec a démontré que les ammocètes, et notamment celles de la Lamproie du Nord, sont aussi appropriées que les mollusques bivalves adultes comme baromètres des niveaux de contaminants organochlorés (Renaud et al. 1995b). Elles sont considérées comme un bon bio-indicateur de la contamination au mercure en plus de pouvoir indiquer les différences dans les niveaux de contamination en métaux entre les rivières (Renaud et al. 1998).

\section{Bilan de la situation}

Taille des populations et tendances démographiques

Aucune estimation de la taille totale des populations de la Lamproie du Nord n'est disponible pour le Québec ou ailleurs dans son aire de répartition (COSEPAC 2007*). On ne connaît donc pas sa situation exacte ni la tendance démographique des populations identifiées à ce jour. Seules des données ponctuelles de taille de populations sont disponibles, lesquelles sont présentées ci-après.

À Saint-Césaire, dans la rivière Yamaska, 61 adultes et 842 ammocètes ont été capturés entre 1946 et 1950, dont 721 ammocètes entre le 27 juillet et le 30 août 1949, au cours d'une étude menée par Vladykov (1952). En dépit d'un effort de pêche intensif, aucun spécimen n'a été capturé au même endroit en 1990 et 1992 (Renaud et al. 1995a; C. B. Renaud, non publié). L'état actuel de l'espèce dans l'ensemble de la rivière reste à préciser.

Deux individus métamorphosés et plusieurs ammocètes ont été recensés dans la rivière Saint-François près de Pierreville entre 1947 et 1951 (Vladykov 1952). Une étude réalisée en 1990 a permis la récolte de deux ammocètes dans cette localité, près de l'île Cartier (Renaud et al. 1995a). L'espèce était donc toujours présente à l'époque, bien qu'aucune donnée ne soit disponible quant à l'abondance de la population. La situation actuelle à cette localité demeure inconnue. Toutefois, l'espèce fut de nouveau observée dans cette rivière le 26 août 2003 à Bromptonville, soit à $130 \mathrm{~km}$ en amont de la localité de Pierreville (Desroches et al. 2008). Il s'agissait d'une ammocète vivante trouvée dans un bassin. De même, environ douze ammocètes mortes et deux vivantes ont été observées le 20 septembre 1995 dans la rivière Massawippi, un tributaire de la rivière Saint-François, à North Hatley (Desroches et al. 2008). Quelques secteurs du bassin versant de la rivière Saint-François semblent donc propices à la Lamproie du Nord.

Une seule femelle adulte a été capturée dans la rivière Gatineau en 1999 (Comtois et al. 2004). Un seul individu a aussi été capturé dans la rivière aux Outardes Est en 2002 (Jean Dubé, communication personnelle). La mention de la localité des Rapides de Lachine concerne six ammocètes capturées le 26 juillet 1950 alors que celle du bassin de la rivière Nicolet (rivière Nicolet Sud-Ouest) se rapporte à une ammocète notée le 22 juillet 1951 (Vladykov 1952). Respectivement trois et six individus de sexe et de stade indéterminé ont été rapportés sur les rivières Trout et Hinchinbrooke en 1976 par le Centre de données sur le patrimoine naturel du Québec (CDPNQ) et le ministère des Ressources naturelles et de la Faune du Québec, ainsi qu'un adulte en 1998 dans la rivière des Prairies par le CDPNQ. Finalement, la mention de 1990 de la rivière Richelieu concerne cinq ammocètes (Renaud et al. 1995b).

\section{Menaces à la survie de l'espèce au Québec}

La détérioration de l'habitat et la pollution des eaux semblent représenter les principales menaces à la survie des lamproies (Renaud 1997; Moyle et Cech 2004), incluant la Lamproie du Nord. La pollution de la rivière Yamaska, associée à une agriculture intensive et à des rejets industriels et urbains, apparaît comme une cause possible de l'absence de mention de l'espèce dans la localité de Saint-Césaire en 1990 et 1992 (Renaud et al. 1995a; C. B. Renaud, non publié). L'utilisation intensive de l'herbicide atrazine appliqué dans la culture du maïs serait également en cause. Lessivé dans la rivière suite aux fortes pluies, ce produit chimique réduirait de façon importante le phytoplancton dont se nourrissent les ammocètes (Renaud et al. 1995a). On retrouve également de nombreux autres pesticides dans la rivière Yamaska et plusieurs de ses tributaires, dont le métolachlore, la cyanazine et le dicamba (Gouvernement du Québec 1998*). Il n'est pas rare de découvrir plus de six pesticides différents au même endroit. La prédominance de la culture du maïs qui nécessite régulièrement l'application de ces produits expliquerait en bonne partie cette situation. Malgré tous les efforts qui ont été déployés au cours des dernières années, la rivière Yamaska présente toujours la pire qualité d'eau au Québec. Le portrait n'est pas plus réjouissant dans le secteur particulier de SaintCésaire (Gouvernement du Québec 1998*).

D'autres rivières où l'espèce fut observée telles que les rivières Richelieu et Nicolet, sont aussi d'une qualité relativement mauvaise (Gouvernement du Québec 1993*, 1995*). En fait, depuis 1992, le ministère du Développement durable, de l'Environnement et des Parcs mène un programme de suivi annuel des 
pesticides dans quatre cours d'eau des régions agricoles où la culture du maïs est importante (Gouvernement du Québec, 2006*). Ces sont les rivières Chibouet (bassin de la rivière Yamaska), des Hurons (bassin de la rivière Richelieu), Saint-Régis (affluent direct du fleuve Saint-Laurent) et Saint-Zéphirin (bassin de la rivière Nicolet). Des pesticides ont régulièrement été détectés durant l'été dans ces rivières. Les substances trouvées le plus souvent sont l'atrazine, le métolachlore, le bentazone, le dicamba, le 2,4-D et le diméthénamide. Ces substances représentent autant de menaces pour la Lamproie du Nord en affectant la qualité de l'eau.

Une autre menace importante à la survie de la Lamproie du Nord au Québec est la disparition des bandes riveraines qui provoque l'érosion des sols et augmente la sédimentation dans les lits des cours d'eau, altérant ainsi les aires de fraie. Ce phénomène constitue une menace importante pour les lamproies (Moyle et Cech 2004). Les bandes riveraines boisées, par leur rôle de filtres et de stabilisateurs des berges, protègent aussi les cours d'eau contre les apports en fertilisants et en pesticides (Société de la faune et des parcs du Québec 2003*). Elles procurent aussi de l'ombre, élément important de l'habitat des ammocètes (Potter et al. 1986). Les superficies requises pour épandre les surplus de lisier de porc et les monocultures comme celle du maïs sont, entre autres, responsables de la disparition des bandes riveraines (Société de la faune et des parcs du Québec 2003*). Les régions de la Montérégie, du Centre-du-Québec et de Chaudière-Appalaches sont particulièrement touchées à cet égard.

La croissance de l'industrie porcine a provoqué une surcharge de fumier et de ses substances polluantes. L'excès de fertilisant, et tout particulièrement le phosphore, touche directement la qualité de l'habitat de la Lamproie du Nord via l'eutrophisation des cours d'eau (Société de la faune et des parcs du Québec 2003*). Cette menace touche potentiellement les cours d'eau de la Montérégie, du Centre-du-Québec et de ChaudièreAppalaches, lesquelles ont été particulièrement touchées par la production porcine au cours des dernières décennies, comme par exemple les rivières Nicolet, Richelieu, Saint-François et Yamaska.

D'autres menaces potentielles pour l'habitat de la Lamproie du Nord concernent le redressement des petits cours d'eau, lequel pourrait endommager l'habitat utilisé par les adultes reproducteurs, ainsi que la présence d'obstacles (ex. barrages) à la libre circulation de l'espèce entre ses habitats de reproduction et de développement des ammocètes.

\section{Statut actuel et protection légale}

La Lamproie du Nord a été identifiée au Québec comme espèce susceptible d'être désignée «menacée ou vulnérable » en vertu de la Loi sur les espèces menacées ou vulnérables. Au Canada, la Lamproie du Nord figure à l'annexe 3 de la Loi sur les espèces en péril à titre d'espèce "préoccupante », désignation donnée par le COSEPAC en 1991 et une mise à jour a été effectuée en 2007 (COSEPAC 2007*). En tant qu'espèce de poissons, la Lamproie du Nord est protégée au Québec en vertu de la Loi sur la conservation et la mise en valeur de la faune. L'habitat de l'espèce n'est protégé au Québec que sur les terres publiques par l'application du Règlement sur les habitats fauniques, de même qu'à l'échelle fédérale, tant sur les terres privées que sur les terres publiques, en vertu de la Loi sur les pêches et la Politique de gestion de l'habitat du poisson. Au Québec, la Loi sur la qualité de l'environnement et la Politique de protection des rives, du littoral et des plaines inondables s'appliquent également à l'habitat de l'espèce autant en terres publiques que privées.

\section{Évaluation}

Il existe un urgent besoin de réaliser des travaux d'inventaire spécifiques et d'effectuer un suivi des populations de Lamproie du Nord au Québec, en donnant priorité aux sites actuellement connus. Des vérifications s'imposent pour savoir si l'espèce a survécu dans ces cours d'eau et, le cas échéant, si ces populations et leurs habitats sont en bonne santé. Une estimation de la taille des populations serait aussi souhaitable. Cette initiative devrait être considérée comme prioritaire dans le contexte de la conservation de l'espèce au Québec. Actuellement, on estime que $55 \%$ des espèces de lamproies de l'hémisphère nord sont en difficulté (Renaud 1997).

Nos connaissances actuelles sur la situation de la Lamproie du Nord au Québec vis-à-vis sa répartition et l'état de ses populations ne nous permettent pas de nous prononcer quant à la catégorie de conservation à attribuer à cette espèce. En effet, contrairement à sa situation dans la région des Grands Lacs où l'effort de pêche est connu à cause du programme de contrôle de la Lamproie marine, Petromyzon marinus, permettant ainsi de déterminer les tendances des populations de toutes les espèces de lamproies de cette région, les mentions de la Lamproie du Nord au Québec ne sont pas le résultat d'un suivi de longue durée mais plutôt la conséquence de travaux d'inventaire ichtyologique d'ordre généraux (e.g. Comtois et al. 2004). De plus, ce n'est que tout récemment (Desroches et al. 2008) que la présence de la Lamproie du Nord est connue bien en amont de la rivière Saint-François, à environ $130-165 \mathrm{~km}$ de la localité originale de Pierreville (Vladykov 1952), une indication de notre manque d'inventaire à l'échelle de la province. COSEPAC $(2007 *)$ attribua à la Lamproie du Nord de la région des Grands Lacs et du haut Saint-Laurent le statut d'espèce « préoccupante » en raison des menaces dues à l'épandage de larvicides sur environ 50\% de son aire de répartition (i.e. la région des Grands Lacs). Nous souhaitons donc que notre article serve de point de référence pour des études de suivi sur la Lamproie du Nord au Québec et lançons un appel pour celles-ci. 


\section{Remerciements}

Nous remercions J. Dubé (ministère des Ressources naturelles et de la Faune) pour sa contribution de données inédites ainsi qu' Alain Chouinard et Marie-France La Rochelle pour la réalisation de la carte.

Documents cités (identifiés par un * dans le texte)

COSEPAC. 2007. Évaluation et rapport de situation du COSEPAC sur la Lamproie du Nord (Ichthyomyzon fossor) (populations des Grands Lacs - du haut Saint-Laurent et population de la Saskatchewan - Nelson) au Canada Mise à jour. Comité sur la situation des espèces en péril au Canada, Ottawa, Ontario, Canada. vi +34 pages.

Fortin, C., I. Cartier, et M. Ouellet. 2005. Rapport sur la situation de la Lamproie du Nord (Ichthyomyzon fossor) au Québec. Ministère des Ressources naturelles et de la Faune, Direction du développement de la faune, Québec, Québec, Canada. 23 pages.

Gouvernement du Québec. 1993. Qualité des eaux de la rivière Richelieu, 1979 à 1992. Ministère de l'Environnement du Québec, Québec, Québec, Canada. 12 pages.

Gouvernement du Québec. 1995. Qualité des eaux de la rivière Nicolet, 1979 à 1994. Ministère de l'Environnement et de la Faune du Québec, Québec, Québec, Canada. 8 pages.

Gouvernement du Québec. 1998. État de l'écosystème aquatique du bassin versant de la rivière Yamaska. Synthèse 1998. Ministère de l'Environnement et de la Faune du Québec, Québec, Québec, Canada. 12 pages.

Gouvernement du Québec. 2006. L'utilisation des pesticides dans le maïs et le soya. [En ligne] [http://www.menv.gouv. qc.ca/pesticides/mais_soya]

Lanteigne, J. 1991. Status report of the Northern Brook Lamprey, Ichthyomyzon fossor. Report to the Committee on the Status of Endangered Wildlife in Canada (COSEWIC). Canadian Wildlife Service, Ottawa, Ontario, Canada. 17 pages.

Morman, R. H. 1979. Distribution and ecology of lampreys in the lower peninsula of Michigan 1957-75. Great Lakes Fishery Commission. Technical Report (33). Ann Arbor, Michigan, USA. 59 pages.

Société de la faune et des parcs du Québec. 2003. Évaluation des répercussions économiques de la production porcine sur la faune et ses habitats. Vice-présidence au développement et à l'aménagement de la faune, Québec, Québec, Canada. 26 pages.

Vladykov, V. D. 1949. Québec lampreys (Petromyzonidae). List of species and their economical importance. Contribution (26), Department of Fisheries, Québec, Québec, Canada. 67 pages.

Vladykov, V. D., et E. Kott. 1979b. Liste des lamproies (Petromyzonidae) de l'hémisphère nord et leur distribution. Publication diverse spéciale 42. Ministère des Pêches et des Océans, Ottawa, Ontario, Canada. 30 pages.

\section{Littérature citée}

Anderson, A. A., et A. M. White. 1988. Habitat selection of Ichthyomyzon fossor and Lampetra appendix in a northeastern Ohio stream. Ohio Journal of Science 88: 7.

Beamish, F. W. H., et S. Lowartz. 1996. Larval habitat of American Brook Lamprey. Canadian Journal of Fisheries and Aquatic Sciences 53: 693-700.

Cochran, P. A., et A. P. Gripentrog. 1992. Aggregation and spawning by lampreys (genus Ichthyomyzon) beneath cover. Environmental Biology of Fishes 33: 381-387.
Comtois, A., F. Chapleau, C. B. Renaud, H. Fournier, B. Campbell, et R. Pariseau. 2004. Inventaire printanier d'une frayère multispécifique : l'ichtyofaune des rapides de la rivière Gatineau, Québec. Canadian Field-Naturalist 118: 521-529.

Davis, W. A. 1970. The comparative susceptibility of three genera of larval lampreys to a lampricide. M.Sc. thesis, University of Guelph, Guelph, Ontario, Canada. 52 pages.

Desroches, J.-F., D. Pouliot, I. Picard, et R. Laparé. 2008. Nouvelles mentions pour six espèces de poissons d'eau douce rares au Québec. Le Naturaliste canadien 132 (2): $62-66$

Forey, P., et P. Janvier. 1993. Agnathans and the origin of jawed vertebrates. Nature 361: 129-134.

Hardisty, M. W. 1961. Studies on an isolated spawning population of Brook Lampreys (Lampetra planeri). Journal of Animal Ecology 30: 339-355.

Hardisty, M. W., et I. C. Potter. 1971a. The behaviour, ecology and growth of larval lampreys. Pages 85-125 in The biology of lampreys. Volume 1. Sous la drection de M. W. Hardisty et I. C. Potter. Academic Press, New York, New York, USA.

Hardisty, M. W., et I. C. Potter. 1971b. The general biology of adult lampreys. Pages 127-206 in The biology of lampreys. Volume 1. Sous la direction de M. W. Hardisty et I. C. Potter. Academic Press, New York, New York, USA.

Hill, B. J., et I. C. Potter. 1970. Oxygen consumption in ammocoetes of the lamprey Ichthyomyzon hubbsi Raney. Journal of Experimental Biology 53: 47-57.

Hubbs, C. L., et M. B. Trautman. 1937. A revision of the lamprey genus Ichthyomyzon. University of Michigan, Museum of Zoology, Miscellaneous Publications 35, Ann Arbor, Michigan, USA. 109 pages.

Jyrkkanen, J., et D. G. Wright. 1979. First record of the Northern Brook Lamprey, Ichthyomyzon fossor, in the Nelson River drainage, Manitoba. Canadian Field-Naturalist 93: 199-200.

Lanteigne, J. 1981. The taxonomy and distribution of the North American lamprey genus Ichthyomyzon. M.Sc. thesis, University of Ottawa, Ottawa, Ontario, Canada. 150 pages.

Lanteigne, J. 1988. Identification of lamprey larvae of the genus Ichthyomyzon (Petromyzontidae). Environmental Biology of Fishes 23: 55-63.

Lanteigne, J. 1992. Status of the Northern Brook Lamprey, Ichthyomyzon fossor, in Canada. Canadian Field-Naturalist 106: 7-13.

Leach, W. J. 1940. Occurrence and life history of the Northern Brook Lamprey, Ichthyomyzon fossor, in Indiana. Copeia 1940: 21-34.

Manion, P. J., et L. H. Hanson. 1980. Spawning behavior and fecundity of lampreys from the upper three Great Lakes. Canadian Journal of Fisheries and Aquatic Sciences 37: 1635-1640.

Moore, J. W., et J. M. Mallatt. 1980. Feeding of larval lamprey. Canadian Journal of Fisheries and Aquatic Sciences 37: 1658-1664.

Morman, R. H., D. W. Cuddy, et P. C. Rugen. 1980. Factors influencing the distribution of Sea Lamprey (Petromyzon marinus) in the Great Lakes. Canadian Journal of Fisheries and Aquatic Sciences 37: 1811-1826.

Moyle, P. B., et J. J. Cech, Jr. 2004. Fishes. An introduction to ichthyology. Prentice Hall, Upper Saddle River, New Jersey, USA. 744 pages.

Okkelberg, P. 1922. Notes on the life-history of the Brook Lamprey Ichthyomyzon unicolor. Occasional Papers of 
the Museum of Zoology, University of Michigan 125: 114.

Potter, I. C. 1980. Ecology of larval and metamorphosing lampreys. Canadian Journal of Fisheries and Aquatic Sciences 37: 1641-1657.

Potter, I. C., R. W. Hilliard, J. S. Bradley, et R. J. McKay. 1986. The influence of environmental variables on the density of larval lampreys in different seasons. Oecologia 70: 433-440.

Purvis, H. A. 1970. Growth, age at metamorphosis, and sex ratio of Northern Brook Lamprey in a tributary of southern Lake Superior. Copeia 1970: 326-332.

Reighard, J., et H. Cummins. 1916. Description of a new species of lamprey of the genus Ichthyomyzon. Occasional Papers of the Museum of Zoology, University of Michigan 31: $1-12+2$ plates.

Renaud, C. B. 1997. Conservation status of northern hemisphere lampreys (Petromyzontidae). Journal of Applied Ichthyology 13: 143-148.

Renaud, C. B., K. L. E. Kaiser, et M. E. Comba. 1995a. Historical versus recent levels of organochlorine contaminants in lamprey larvae of the St. Lawrence River basin, Québec. Canadian Journal of Fisheries and Aquatic Sciences 52: 268-275.

Renaud, C. B., K. L. E. Kaiser, M. E. Comba, et J. L. Metcalfe-Smith. 1995b. Comparison between lamprey ammocoetes and bivalve molluscs as biomonitors of organochlorine contaminants. Canadian Journal of Fisheries and Aquatic Sciences 52: 276-282.

Renaud, C. B., H. K. T. Wong, et J. L. Metcalfe-Smith. 1998. Trace metal levels in benthic biota from four tributaries to the St. Lawrence River, Québec. Water Quality Research Journal of Canada 33: 595-610.

Schuldt, R. J., et R. Goold. 1980. Changes in the distribution of native lampreys in Lake Superior tributaries in response to Sea Lamprey (Petromyzon marinus) control, 1953-77. Canadian Journal of Fisheries and Aquatic Sciences 37: 1872-1885.
Scott, W. B., et E. J. Crossman. 1974. Poissons d'eau douce du Canada. Bulletin $n^{\circ} 184$. Office des recherches sur les pêcheries du Canada. Environnement Canada, Service des pêches et des sciences de la mer, Ottawa, Ontario, Canada. 966 pages.

Smith, B. R., et J. J. Tibbles. 1980. Sea Lamprey (Petromyzon marinus) in Lakes Huron, Michigan, and Superior: history of invasion and control, 1936-78. Canadian Journal of Fisheries and Aquatic Sciences 37: 1780-1801.

Sutton, T. M., et S. H. Bowen. 1994. Significance of organic detritus in the diet of larval lampreys in the Great Lakes basin. Canadian Journal of Fisheries and Aquatic Sciences 51: 2380-2387.

Trautman, M. B. 1981. The fishes of Ohio. Ohio State University Press, Columbus, Ohio, USA. 782 pages.

Uhland, F. C., I. Mikaelian, et D. Martineau. 2000. Maladies des poissons d'eau douce du Québec : guide de diagnostic. Les Presses de l'Université de Montréal, Montréal, Québec, Canada. 466 pages.

Vladykov, V. D. 1951. Fecundity of Québec lampreys. Canadian Fish Culturist 10: 1-14.

Vladykov, V. D. 1952. Distribution des lamproies (Petromyzonidae) dans la province de Québec. Le Naturaliste canadien 79: 85-120.

Vladykov, V. D. 1973. North American nonparasitic lampreys of the family Petromyzonidae must be protected. Canadian Field-Naturalist 87: 235-239.

Vladykov, V. D., et E. Kott. 1979a. Satellite species among the holarctic lampreys (Petromyzonidae). Canadian Journal of Zoology 57: 860-867.

Wilson, K. A., et K. Ronald. 1967. Parasite fauna of the Sea Lamprey (Petromyzon marinus von Linné) in the Great Lakes region. Canadian Journal of Zoology 45: 1083-1092.

Received 8 March 2006

Accepted 20 August 2008 